\section{A New Active Constituent of Pyrethrum Flower}

THE pyrethrin content as determined by the chemical process is the only factor that influences the market value of pyrethrum, as it is held that a close relationship exists between the amount of pyrethrin present and the number of insects killed. The results of our experiments (see graph) carried out with different samples of pyrethrum, clearly indicate that (1) the percentage kill of insects bears no direct relation to the amount of pyrethrin present in the samples, (2) a smaller amount of pyrethrin extracted from a sample yielding a high pyrethrin value produces better biological results than a larger amount from a poorer sample.

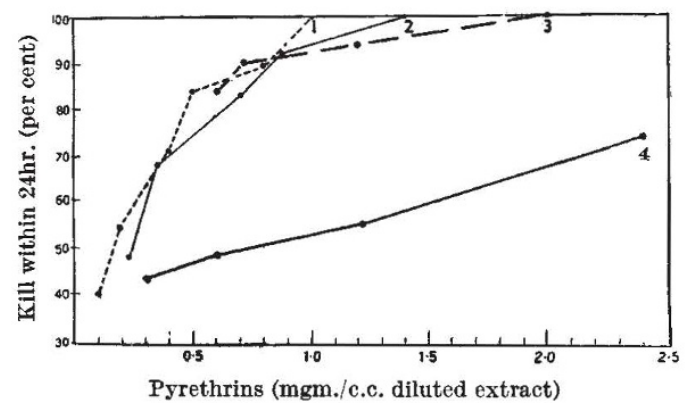

1. Pyrocide 20. Proprietary extract.

2. PREPARED FROM Chrysanthemum cineraricefolium (INDIAN);

PYRETHRIN CONTENT, 0.702 PER CENT.

3. ", " C. cineraricefolium (JAPANESE) ; PYRETHRIN

CONTENT, 0.602 PER CENT.

4. , " C. roseum (INDIAN); PYRETHRIN CONTENT, 0.247 PER CENT.

It therefore follows from the above that in addition to pyrethrins I and II, the only active constituents so far known, there exists some other toxic principle which operates in the same way as pyrethrin in the production of the characteristic effect of pyrethrum on insects. As the nature of this new substance is not known, it may be designated as $X$. Why a smaller quantity of pyrethrin from a rich sample of pyrethrum produces better results than even a considerably larger quantity extracted from poorer samples may be explained by the fact that the active principle $X$ exists in larger amount in the former than in the latter. This also accounts for the fact that a sample of pyrethrum possessing a high pyrethrin content yields better results than another with a lower pyrethrin value.

The results also suggest that the action of the new active principle $X$ is not complementary to that of pyrethrin. It is probably an independent body, the growth of which in the flowers is regulated by the same factors as pyrethrin. Both, however, are soluble in kerosene.

The present work confirms our previous finding that kerosene extract prepared from the sun-dried residue left after the removal of pyrethrins I and II from a sample of pyrethrum flowers was found still active, and this led us to arrive at the tentative conclusion that in addition to pyrethrins there were other substances which were responsible for the destruction of insects and mosquito larvæ ${ }^{1}$.

For each experiment a hundred flies, Musca vicina, all three to five days old, were employed, and our results represent the average of three observations carried out on successive days. The extracts were prepared for us according to the method used for chemical assay ${ }^{2}$. Kerosene was used as the vehicle for diluting the extract; this in itself has not been shown to possess any appreciable insecticidal properties.

The biological variation from the mean calculated from the results of three experiments ranged between 0 and 5 per cent in each instance.

Sample (1) is a commercial standardized preparation known as Pyrocide 20. D. N. Roy.

Department of Medical Entomology,

S. M. Ghosh.

School of Tropical Medicine, Calcutta. April 21.

${ }^{1}$ Chopra, Roy and Ghosh, J. Mal. Inst. Ind., 3, 457 (1940).

'Lahiri, Ghosh and Chopra, $J$ Amer. Pharm. Assoc., 30, 72 (1941).

\section{A Recording Paper for Field and General Use}

Autographic instruments frequently use a recording paper coated with metallic oxides, on which a black trace is made by a style of copper or silver. While this paper is very convenient, it is not always suitable for field work. To meet the need for a recording paper that would withstand difficult conditions, a special paper has been developed at the Road Research Laboratory. The following details of the process are given in the hope that they may be of use to other workers.

A good quality thin tracing paper, having a smooth surface, is damped and lapped round the recorder drum, the overlap of the paper being fixed with ordinary gum. On drying, the paper shrinks and stretches tightly round the drum, giving a very firm surface with a scarcely perceptible joint. When dry, the paper is spray-painted with a suspension of zine oxide in dilute cellulose varnish. The resulting surface is pure white in colour, with a smooth matt texture, and on it a hard bronze style makes an excellent black trace.

Drums so coated can be used up to a very high writing speed; a time-base of 300 in. per sec. has been used with complete success, and there is no indication that higher speeds could not be used. The paper surface is strong enough to withstand the style running many times over the same base line without the paper being cut, and the hard bronze style keeps its point very satisfactorily.

In particular, the paper is waterproof, in the sense that rain falling on the paper can be wiped off without affecting the surface. Indeed, records have sometimes been irrecoverable for some days after use, during which period they have been exposed to rain with no damage to the record. It is not desirable to expose the paper to prolonged damp before use, as ultimately the paper absorbs water, swells, and becomes slack on the drum, but an accidental splash does not matter. When damp conditions are likely to be met, the cellulose coating should be rather thicker than is normally used.

After use, the paper is removed from the drum by slitting it with a sharp knife: if the paper is then dry it will come away cleanly from the drum. The resulting records are fully permanent, and will withstand any ordinary handling, filing, etc. They can be written on with pen or pencil, and pencil marks can be erased as with any ordinary paper. The sheets are also sufficiently translucent to allow records to be compared by superposition, or to be measured by putting them over a sheet of strongly marked squared paper. 